\title{
Supporting Information: Predictive theoretical framework for dynamic control of bio-inspired hybrid nanoparticle self-assembly
}

Xin Qi ${ }^{\dagger}$ Yundi Zhao, ${ }^{\dagger}$ Kacper Lachowski, ${ }^{\dagger} \ddagger$ Julia Boese, ${ }^{\dagger}$ Yifeng Cai, $^{\dagger}$ Orion Dollar, ${ }^{\dagger}$ Brittney Hellner, ${ }^{\dagger}$ Lilo Pozzo, ${ }^{\dagger}$ Jim Pfaendtner, ${ }^{\dagger}, \boldsymbol{\Phi}$ Jaehun Chun, ${ }^{*}, \boldsymbol{\Phi}, \S$ François Baneyx, ${ }^{*, \dagger}$ and Christopher J. Mundy*, $\mathbf{\uparrow}, \dagger$

$\dagger$ Department of Chemical Engineering, University of Washington, Seattle, 98195

Washington, United States

$\ddagger$ Molecular Engineering and Sciences Institute, University of Washington, Seattle, 98195

Washington, United States

IPhysical and Computational Sciences Directorate, Pacific Northwest National Laboratory, Richland, Washington 99354, United States

$\S$ Levich Institute and Department of Chemical Engineering, CUNY City College of New York, New York, New York 10031, United States

E-mail: jaehun.chun@pnnl.gov; baneyx@uw.edu; chris.mundy@pnnl.gov 


\section{Free energies of adsorption from SPR measurements}

We use a two-step cooperative model that accurately captures the cooperative nature of the adsorption of Car9 derivatives to silica to simultaneously fit sigmoidal sensorgrams acquired at two different protein concentrations. ${ }^{1}$ The kinetic parameters are extracted and the binding free energy calculated as described. ${ }^{2}$ For the sfGFP::Car9 loop variant the $\Delta G$ is -43.6 $\mathrm{kJ} / \mathrm{mol}$ at $\mathrm{pH} 7.5$ (Figure $\mathrm{S} 1$ ). This value is $20 \%$ lower than the $\Delta \mathrm{G}$ of $-53.1 \mathrm{~kJ} / \mathrm{mol}$ that we previously reported for the tail-tagged variant, ${ }^{2}$ and consistent with a lower affinity of sfGFP::Car9 for silica relative to sfGFP-Car9 (c.f., Figure 1(b) in the main text).

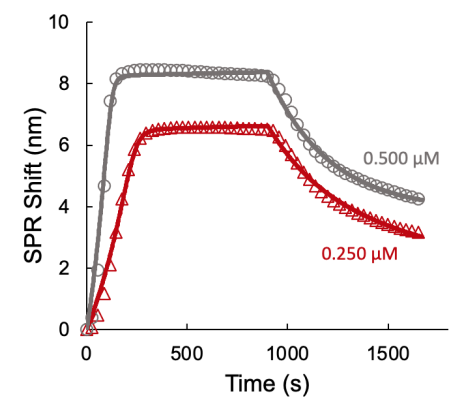

\begin{tabular}{|c|c|c|c|c|c|c|c|c|c|c|c|c|}
\hline Protein & $u$ & $\begin{array}{c}k_{\mathrm{a} 1}^{\prime} \\
\left(\mathrm{M}^{-1} \mathrm{~S}^{-1}\right)\end{array}$ & $\begin{array}{c}k_{\mathrm{d} 1} \\
\times 10^{-3}\left(\mathrm{~s}^{-1}\right) \\
\end{array}$ & $\begin{array}{c}k_{\mathrm{a} 2} \\
\times 10^{-3}\left(\mathrm{~s}^{-1}\right) \\
\end{array}$ & $\begin{array}{c}k_{\mathrm{d} 2} \\
\times 10^{-3}\left(\mathrm{~s}^{-1}\right) \\
\end{array}$ & $\theta$ & $\begin{array}{c}k_{\mathrm{a} 1} \\
\times 10^{-3}\left(\mathrm{~s}^{-1}\right) \\
\end{array}$ & $K_{\mathrm{d} 1}$ & $K_{\mathrm{d} 2}$ & $\begin{array}{c}\Delta \mathrm{G}_{1} \\
\mathrm{~kJ} \cdot \mathrm{mol}^{-1}\end{array}$ & $\begin{array}{c}\Delta \mathrm{G}_{2} \\
\mathrm{~kJ} \cdot \mathrm{mol}^{-1}\end{array}$ & $\begin{array}{c}\Delta \mathrm{G} \\
\mathrm{kJ} \cdot \mathrm{mol}^{-1}\end{array}$ \\
\hline sfGFP-Car9 & 2.9 & 20700 & 0.76 & 0.17 & 0.03 & 0.89 & 273454 & 2.7793E-09 & 0.18 & -48.8 & -4.3 & -53.1 \\
\hline sfGFP::Car9 & 2.8 & 10000 & 2.7 & 0.66 & 0.31 & 0.61 & 55179 & 4.8932E-08 & 0.47 & -41.7 & -1.9 & -43.6 \\
\hline
\end{tabular}

Figure S1: Modeling the sigmoidal adsorption kinetics of sfGFP::Car9 (loop-tag Car9) on silica-coated SPR chips. Sensorgrams are collected at the indicated protein concentrations. A wash phase in which protein-free buffer is flowed over the chip starts at $\mathrm{t}=900 \mathrm{~s}$ and causes protein desorption. Solid lines in panels are obtained by fitting the experimental data to a previously described two-step cooperative adsorption model and kinetic parameters are extracted from a simultaneous fit of the two sensorgrams. ${ }^{1}$

\section{Characterization by dynamic light scattering (DLS)}

We probe the reversibility of protein-mediated nanoparticle assembly using size distributions acquired by DLS. We first examine the size of $1 \mu \mathrm{M}$ bare SiNP in $20 \mathrm{mM}$ Tris-HCl buffered at $\mathrm{pH} 7.5$ and 8.5. We find that the mean values for hydrodynamic diameters $\left(D_{h}\right)$ are 17.6 \pm 1.2 and $17.1 \pm 1.3 \mathrm{~nm}$ for $\mathrm{pH} 7.5$ and 8.5, respectively, which are in good agreement 
with the manufacturer'as specifications of a $10 \mathrm{~nm}$ diameter.

Next, we perform a similar characterization on mixtures of protein and SiNP at varying $\mathrm{pH}$ values. Using a 5-fold molar excess of sfGFP::Car9-Car9 over the molar equivalent of SiNP, we show in Figure S2(a) that the assembled state depends on the solution $\mathrm{pH}$ and that a sharp transition occurs between $\mathrm{pH} 7.5$ and 8.0. At $\mathrm{pH}$ 7.0, SiNPs bridged by protein molecules rapidly sediment into large and polydisperse clusters whose size can not be accurately accessed by DLS. At pH 7.5, SiNP settle out of solution after 30 minutes of quiescent incubation and form polydisperse clusters with mean $D_{h} \sim 2000 \mathrm{~nm}$. At pH 8.0, however, we observe a sharp transition to stable $\sim 55 \mathrm{~nm}$ clusters while a further increase of $\mathrm{pH}$ to 8.5 leads to distribution whose mean $D_{h}$ is consistent with that of a protein-coated SiNP singlet ( $24 \mathrm{~nm}$; Figure S2(b)).

(a)

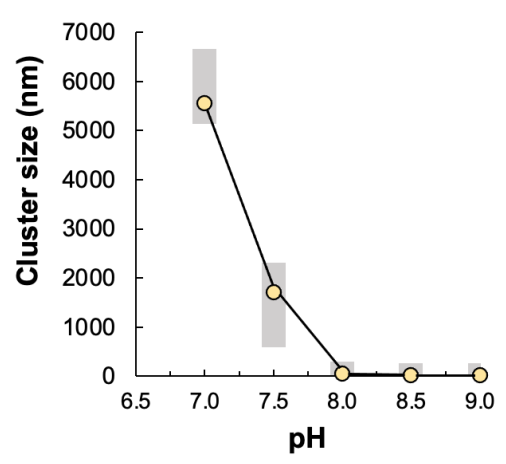

(b)

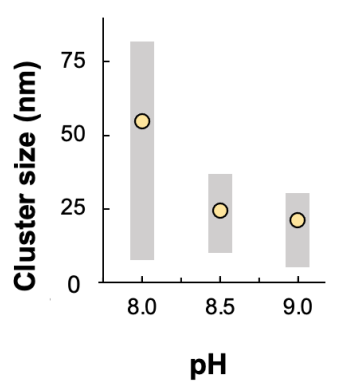

(c)

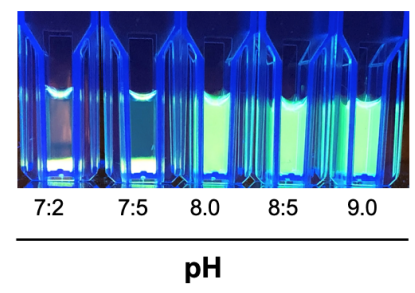

Figure S2: Influence of $\mathrm{pH}$ on size distributions. (a) The measurements of $D h$ for $\mathrm{pH} 7.0$ to 9.0. (b) A magnified view of the measurements of $D h$ for $\mathrm{pH} 8.0$ to 9.0. (c) The appearance of UV-illuminated samples at $\mathrm{pH} 7.2$ to 9.0 after $30 \mathrm{~min}$ of quiescent incubation. The DLS value is unreliable at $\mathrm{pH} 7.0$ but the distribution mean is provided for reference. Gray bars corresponding to the size distributions' full width at half maximum (FWHM) provide a measure of cluster heterogeneity.

\section{FRET characterization}

We develop a new Förster resonance energy transfer (FRET) assay by taking advantage of the spectral overlap between the emission spectrum of sfGFP (donor) and the adsorption 
spectrum of rhodamine molecules encapsulated within the SiNP as described in the legend of Figure S3. FRET spectra are acquired on $500 \mu \mathrm{L}$ samples in a $10 \mathrm{~cm}$ path length quartz cuvette using a F4500 fluorescence spectrophotometer (Hitachi) with an excitation wavelength of $485 \mathrm{~nm}$ (sfGFP), slit widths set at $2.5 \mathrm{~nm}$, and a scan speed of $240 \mathrm{~nm} / \mathrm{min}$. FRET efficiencies are calculated by measuring the change in the donor fluorescence intensity in the absence and presence of acceptor, using:

$$
E=1-\frac{I_{D A}}{I_{D}}
$$

where $I_{D A}$ and $I_{D}$ are the total donor fluorescence intensities in presence and absence of acceptor respectively. ${ }^{3}$

\section{Ultra-small-angle x-ray scattering (USAXS)}

\section{Material and methods}

Rhodamine-conjugated silica nanoparticles (RhSiNP) terminated with surface silanols were purchased from Micromod Partikeltechnologie GmbH (Rostock, Germany). sfGFP::Car9Car9 (sfGFP) was mixed in centrifuge tubes with RhSiNP at a 5:1, 3:1, or 1:1 molar ratio in $20 \mathrm{mM}$ Tris- $\mathrm{HCl}$ titrated to a $\mathrm{pH}$ of 7.5 or 8.5. The mixture was then covered with aluminum foil and rotated using a tube rotator for 30 minutes at room temperature.

SiNP and sfGFP::Car9-Car9 are mixed in centrifuge tubes at a 5:1 prot:SiNP molar ratio in $20 \mathrm{mM}$ Tris- $\mathrm{HCl}$ titrated to a $\mathrm{pH}$ of 7.5 or 8.5 . The mixture is covered with aluminum foil and rotated using a tube rotator for 30 minutes at room temperature.

USAXS data were collected on the 9-ID-C beamline at the Advanced Photon Source at Argonne National Laboratory ${ }^{4}$ using $21 \mathrm{keV}$ X-rays. USAXS data collection time was 90 seconds. Beam size was 0.8 x $0.8 \mathrm{~mm}$. Flux density was approximately $5 \times 10^{12}$ photons $/$ second $/ \mathrm{mm}^{2}$. Samples were loaded in a custom-made 48-well plate assembly with two Kapton windows and a sample thickness of $10 \mathrm{~mm}$. USAXS data were reduced using 
(a)

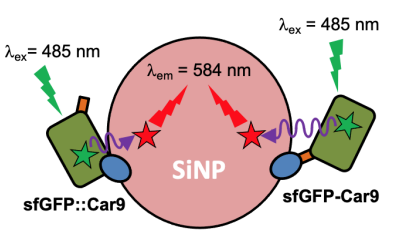

(b)

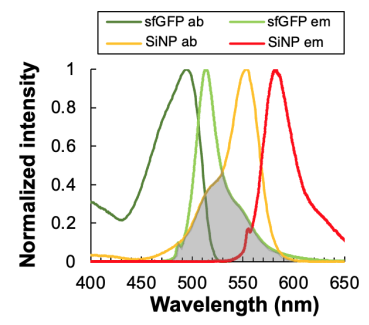

(c)

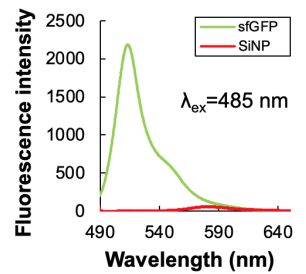

(d)

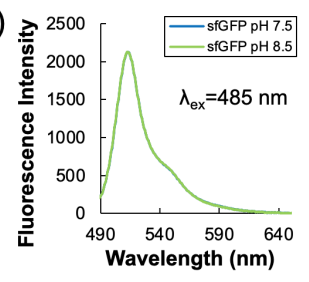

(e)

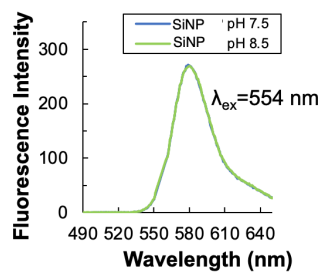

(f)

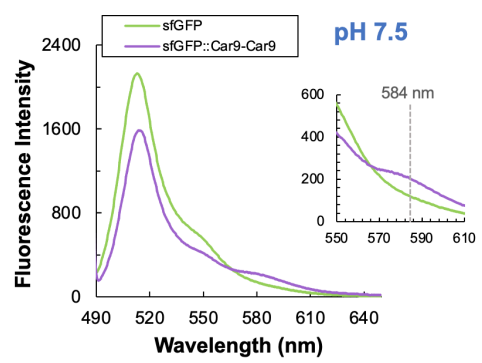

(g)

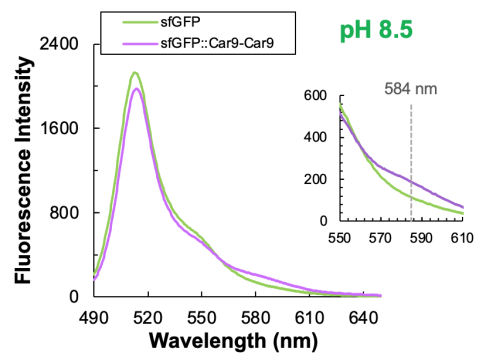

Figure S3: Development of a FRET assay relying on sfGFP as donor and SiNP encapsulating rhodamine dye as an acceptor. (a) A cartoon illustration of absorption and emission in the hybrid nanoparticle system. (b) Normalized absorption (ab) and emission (em) spectra of sfGFP and SiNP. sfGFP has a maximum emission peak at $512 \mathrm{~nm}$ when excited at $485 \mathrm{~nm}$, while SiNP exhibits maximum adsorption at $554 \mathrm{~nm}$ and emits at $584 \mathrm{~nm}$. The shaded region corresponds to the overlap between the emission spectrum of sfGFP and the absorption spectrum of SiNP. (c) Emission spectra of $5 \mu \mathrm{M}$ sfGFP (green) and $1 \mu \mathrm{M}$ SiNP (red) upon $485 \mathrm{~nm}$ excitation spectra in $\mathrm{pH} 7.5$ buffer show there is no cross-talk between acceptor and donor. The emission spectra of the sfGFP donor (d) and of the SiNP acceptor (e) completely overlap when measured in buffer held at $\mathrm{pH} 7.5$ or 8.5, indicating that FRET is not affected in this range of $\mathrm{pH}$. (f-g) FRET emission spectra collected with $5 \mu \mathrm{M}$ of the indicated proteins and $1 \mu \mathrm{M}$ of $10 \mathrm{~nm}$ RhSiNP following excitation at $485 \mathrm{~nm}$ in $20 \mathrm{mM}$ Tris- $\mathrm{HCl}$ solutions held at $\mathrm{pH} 7.5$ or 8.5. Insets show a magnified view of the FRET emission peak at $584 \mathrm{~nm}$.

standard data reduction protocols and software (Indra) that were provided by the beamline. Data were corrected for instrumental background and for the scattering from the sample container and windows. Mathematical desmearing of the USAXS data was performed to remove instrumental slit smearing effects for direct comparison with SAXS profiles obtained from rigid-body simulations. ${ }^{5}$ USAXS data were also analyzed using SASview/SASmodels ${ }^{6,7}$ and bumps ${ }^{8}$ packages for Python. 


\section{USAXS Data Fitting and Analysis}

As shown in Figure 5 in the main text and Figure S4, the USAXS profiles of samples prepared at a $\mathrm{pH}$ of 8.5 at sfGFP:SiNP molar ratios of 1:1 and 3:1 were qualitatively similar to the scattering from fully dispersed SiNP with no protein present at a $\mathrm{pH}$ of 7.5 . This was consistent with the expectation that aggregation of SiNP induced by sfGFP should be minimal at a $\mathrm{pH}$ of 8.5. Therefore, the SiNP suspension and the samples prepared at a $\mathrm{pH}$ of 8.5 with sfGFP:SiNP molar ratios of $1: 1$ and 3:1 were all fitted using a sphere form factor with a lognormal distribution of radii to account for polydispersity. The sphere radius was fixed at $4.7 \mathrm{~nm}$ and the radius polydispersity of the bare SiNP was fitted. This radius and radius polydispersity were then used for all samples. Note that the radius was comparable to that reported by the manufacturer $(5 \mathrm{~nm})$ as seen in Table S1. The remaining USAXS data from aggregated particles were fitted using a fractal aggregate model with core-shell spheres as the building blocks. ${ }^{9,10}$ This model represents the product of the form factor of a coreshell sphere, $P(q)$, and the structure factor of a fractal aggregate to represent inter-particle scattering contributions, $S(q)$ :

$$
\begin{gathered}
I(q)=P(q) S(q)+\text { background }, \\
P(q)=\frac{\phi}{V_{s}}\left[3 V_{c}\left(\rho_{c}-\rho_{s}\right) \frac{\sin \left(q r_{c}\right)-q r_{c} \cos \left(q r_{c}\right)}{\left(q r_{c}\right)^{3}}+3 V_{s}\left(\rho_{s}-\rho_{\text {solvent }}\right) \frac{\sin \left(q r_{s}\right)-q r_{c} \cos \left(q r_{s}\right)}{\left(q r_{s}\right)^{3}}\right], \\
S(q)=1+\frac{D_{f} \Gamma\left(D_{f}-1\right)}{\left[1+1 /(q \xi)^{2}\right]^{\left(D_{f}-1\right) / 2}} \frac{\sin \left[\left(D_{f}-1\right) \tan ^{-1}(q \xi)\right]}{\left(q r_{s}\right)^{D_{f}}}
\end{gathered}
$$

Here, $\phi$ is the volume fraction of particles, $V_{s}$ is the volume of whole particle, $V_{c}$ is the volume of the core of a core-shell particle, $\rho_{c}, \rho_{s}, \rho_{\text {solvent }}$ are the scattering length densities of the core, shell, and solvent, respectively, $r_{c}$ is the radius of core, $r_{s}$ is the radius of total particle, $D_{f}$ is the fractal dimension, and $\xi$ is the correlation length. 


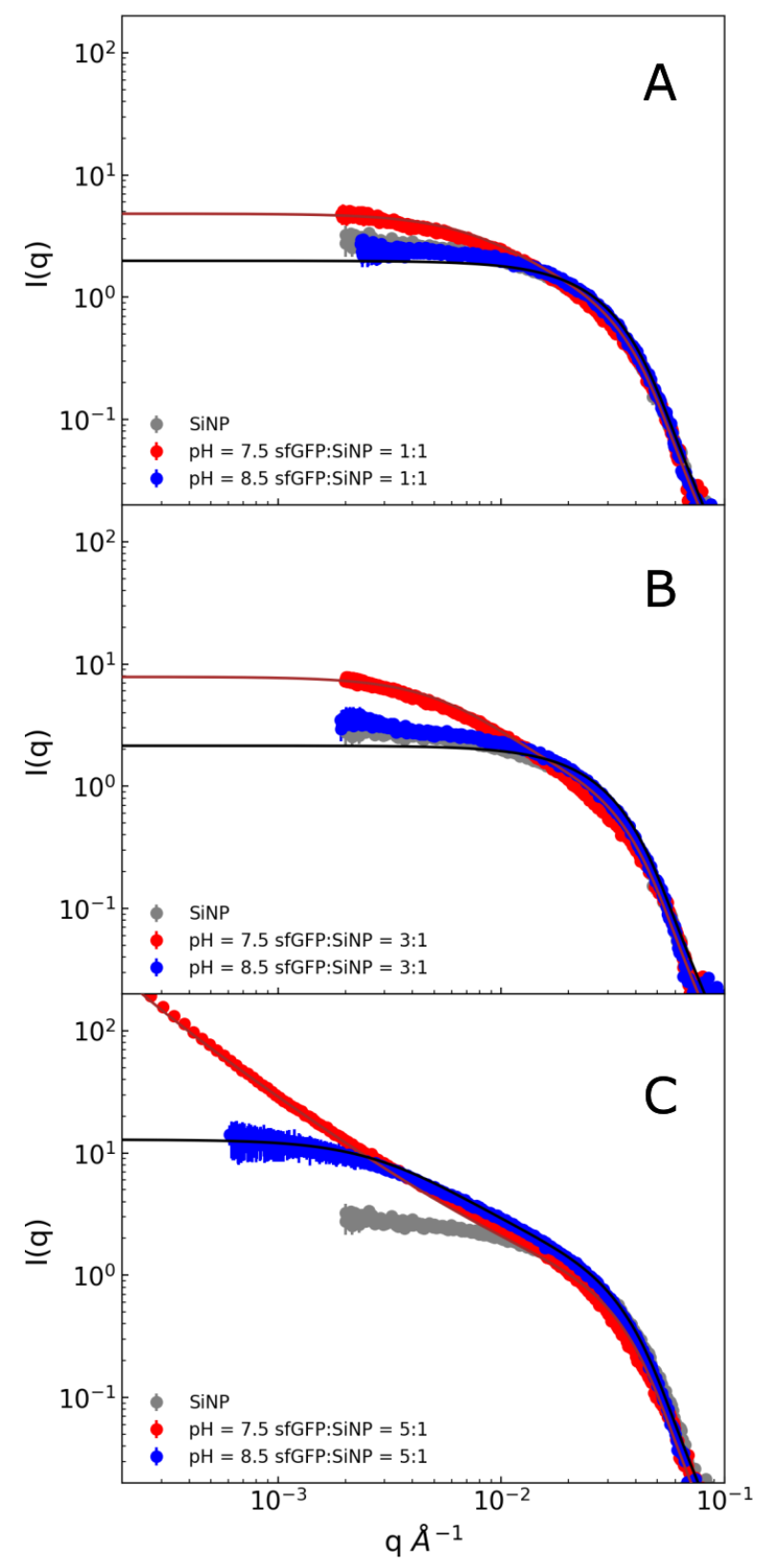

Figure S4: Smeared USAXS data of all sfGFP:SiNP molar fractions and $\mathrm{pH}$ conditions that were characterized. A, B, and C correspond to sfGFP:SiNP molar fractions of 1:1, 3:1, and 5:1, respectively. Scattering from SiNP measured at a $\mathrm{pH}$ of 7.5 at the same particle concentration with no protein present is plotted for reference. Solid lines correspond to the fitted model. 
Table S1: Results of fitting a fractal aggregate model composed of core-shell subunits, or a simple spherical model to all samples.

\begin{tabular}{|c|c|c|c|c|c|c|c|c|c|}
\hline $\mathrm{pH}$ & $\begin{array}{l}\text { sfGFP:SiNP } \\
\text { Molar Ratio }\end{array}$ & $\begin{array}{c}\text { Radius } \\
{[\AA]}\end{array}$ & Radius PD & $\begin{array}{c}\text { Thickness } \\
{[\AA]}\end{array}$ & $\begin{array}{c}\rho_{c} \\
{\left[10^{-6} A^{-2}\right]}\end{array}$ & $\begin{array}{c}\rho_{\mathrm{s}} \\
{\left[10^{-6} \mathrm{~A}^{-2}\right]}\end{array}$ & $\begin{array}{c}\rho_{\text {solvent }} \\
{\left[10^{-6} A^{-2}\right]}\end{array}$ & $D_{f}$ & $\begin{array}{c}\xi \\
{[A]}\end{array}$ \\
\hline 8.5 & 5 & 47.0 & 0.30 & 25 & 16.99 & 9.43 & 9.43 & 2.33 & 308 \\
\hline 8.5 & 3 & 47.0 & 0.38 & & 16.99 & & 9.43 & & \\
\hline 8.5 & 1 & 47.0 & 0.38 & & 16.99 & & 9.43 & & \\
\hline 7.5 & 5 & 47.0 & 0.38 & 25 & 16.99 & 9.43 & 9.43 & 2.25 & 19998 \\
\hline 7.5 & 3 & 47.0 & 0.38 & 25 & 16.99 & 9.43 & 9.43 & 2.99 & 124 \\
\hline 7.5 & 1 & 47.0 & 0.38 & 25 & 16.99 & 9.43 & 9.43 & 2.99 & 91 \\
\hline 7.5 & 0 & 47.0 & 0.30 & & 16.99 & & 9.43 & & \\
\hline
\end{tabular}


In this model, the shell represents the layer of adsorbed protein and water that surrounds each SiNP and that serves as a "spacer" for the assembled particles. Given that the layer surrounding the SiNP is primarily composed of solvent, the X-ray scattering length density (SLD) of the shell is approximated as that of the solvent, $9.43 \times 10^{-6} \AA^{-2}$, whereas the X-ray SLD of the SiNP core is $16.99 \times 10^{-6} \AA^{-2}$. Thus, in the limit of a shell SLD that is equal to the solvent, the form factor of the core-shell sphere form-factor $\mathrm{P}(\mathrm{Q})$ approaches that of a simple sphere. The structure factor on the other hand, remains sensitive to the thickness of the shell due to the interparticle spacing that is introduced between particles that are bridged by sfGFP adsorbed on the surfaces. The thickness of the shells in the model was fixed at 2.5 $\mathrm{nm}$ to account for the spacing of particles bridged by sfGFP, which is approximately $5 \mathrm{~nm}$ in the axial direction when also accounting for the SBPs. The fractal dimension, $D_{f}$, that is obtained from the model describes the distribution of mass inside a fractal. A schematic of the clusters that form between the limits of $1<D_{f}<3$ is shown in Figure S5. For randomly aggregating mass fractals, $D_{f}$ typically varies between approximately 1.5 and 2.5 and is correlated with the density of the distribution of subunits in the cluster. Finally, the correlation length $\xi$ is proportional to the maximum size of the fractal aggregates.
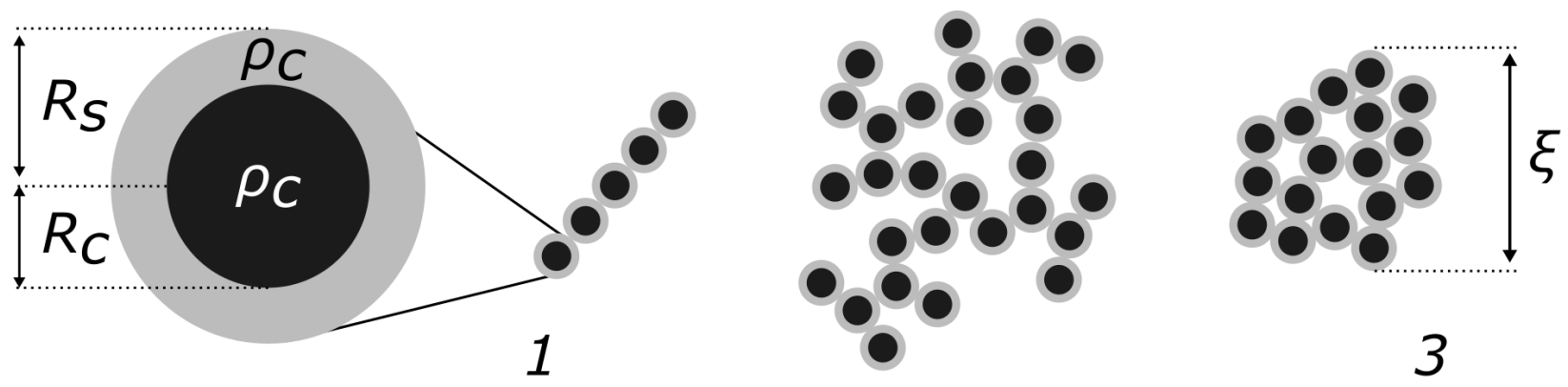

\section{Df}

Figure S5: Schematic showing the model of the core-shell sphere building block on the left, and the fractal aggregate on the right. Note that in the software implementation of the core-shell sphere form factor, the thickness is the difference between $R_{s}$ and $R_{c}$.

Assembly is observed for all sfGFP:SiNP molar fractions which were prepared at a pH of 
7.5. The value of $D_{f}$ can also be linked to the kinetics of aggregation, ${ }^{11}$ where lower values correspond to fast, diffusion-limited aggregation, and conversely higher values correspond to slow, reaction-limited aggregation. The sample prepared at a 5:1 sfGFP:SiNP molar fraction and a $\mathrm{pH}$ of 8.5 also showed aggregation, and had a similar fractal dimension $\left(D_{f}=2.33\right)$ as the sample with the same molar fraction prepared at a $\mathrm{pH}$ of $7.5\left(D_{f}=2.25\right)$. Similarly, whereas increasing the protein concentration led to formation of larger clusters for samples prepared at a $\mathrm{pH}$ of 7.5, the clusters formed at a $\mathrm{pH}$ of 8.5 and 5:1 sfGFP:SiNP molar fraction were smaller $(\xi=308 \AA)$ than those obtained at pH $7.5(\xi>19998 \AA)$. The maximum size of the latter cannot be determined due to a lack of a Guinier region in the q-range of the USAXS instrument.

Together, these results indicate that the kinetics of aggregation and the final structure of the cluster can be controlled by both the $\mathrm{pH}$ and the protein concentration. However, sampling of additional experimental conditions, in-situ USAXS kinetics experiments, and additional simulation efforts will be necessary to yield more predictive control over the final structure of SiNP clusters.

\section{Theory and computation}

\section{Colloidal forces between SiNP}

As given by Eq. 1 in the main text, we use the mean field approach for estimate the long-range interaction between two SiNPs in an aqueous solution, which is composed of an attractive vdW term, $W_{\mathrm{SS}}(r)$, and an electrostatic repulsion term, $V_{\mathrm{SS}}(r)$. For two spheres of equal sizes, the Hamaker theory ${ }^{12}$ predicts

$$
W(r)=-\frac{A}{6}\left(\frac{2 R^{2}}{r^{2}-4 R^{2}}+\frac{2 R^{2}}{r^{2}}+\ln \frac{r^{2}-4 R^{2}}{r^{2}}\right)
$$


Here $A$ is the Hamaker constant $\left(1.02 \times 10^{-20} \mathrm{~J}\right.$ for SiNP, ${ }^{13} r$ is the center-to-center distance between two colloidal particles, and $R$ is the particle radius. By assuming the leading term in Eq. S5 dominates, and that inter-particle interaction is only significant when the separation between two particles is much smaller than $R$ (i.e., $r-2 R \ll R$ ), we arrive at the $\mathrm{vdW}$ dispersion between two SiNPs in an aqueous solution

$$
W_{\mathrm{SS}}(r)=-\frac{A R_{\mathrm{S}}}{12\left(r-2 R_{\mathrm{S}}\right)} .
$$

We use the following figure to justify the assumption that allows us to use Eq. S6 instead of Eq. S5. The scaled energetics of Eq. S5 and S6 with respect to $k_{\mathrm{b}} \mathrm{T}$ in a log plot as shown in Figure S6 shows that the two curves only differ significantly at longer separations, as expected, but the scale of the difference is on the order of $0.01 k_{\mathrm{b}} \mathrm{T}$.

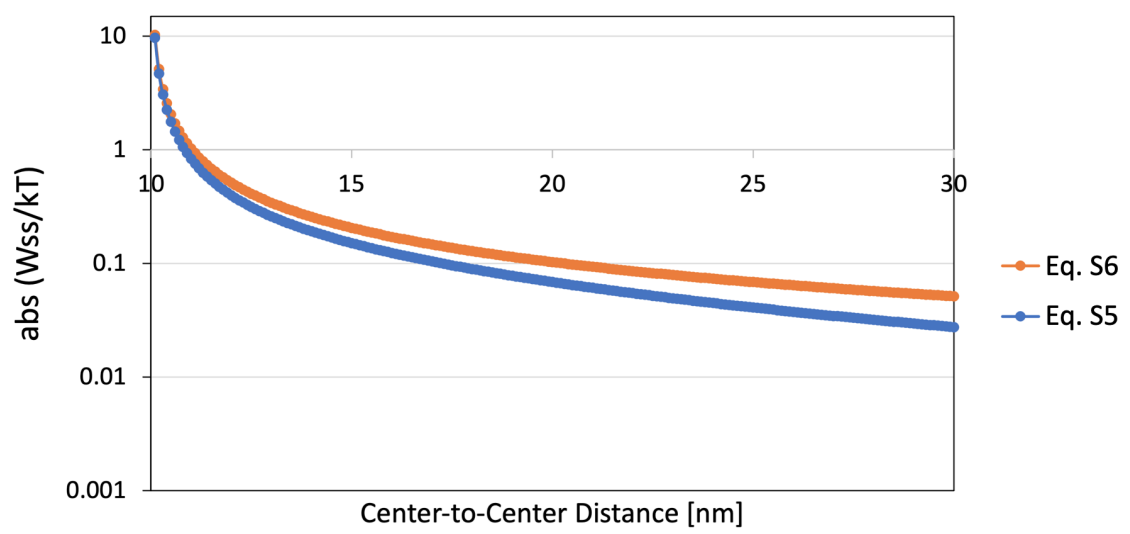

Figure S6: A comparison of the scaled $W_{\mathrm{SS}}$ of Eq. S5 and S6.

The repulsion between charged objects in ionic solution arises from collective phenomena between the SiNP surface and the solution, resulting in a electric double layer. We capture this phenomena using the standard mean-field approach, namely the linearized PoissonBoltzmann equation. With the assumption of a small surface potential $\psi$, and low ionic strength, the linearized Poisson-Boltzmann equation becomes adequate and yields the famil- 
iar Debye-Hückel description the screening of the SiNP charge.

$$
\nabla^{2} \psi-\kappa^{2} \psi=0
$$

Here, $\nabla$ is the Laplacian operator, $\psi$ is the potential at the SiNP surface, and $\kappa$ is the Debye screening parameter.

For two charged spheres of similar sizes at any separation, Sader et al. ${ }^{14}$ derived an analytic formula from Eq. S7 by applying the Derjaguin approximation in conjunction with the proper boundary conditions for $\psi$. With $\psi$ being the absolute electrostatic potential at the particle surface and 0 at the long-range limit, we have

$$
V_{\mathrm{SS}}(r)=\varepsilon\left(\frac{k_{\mathrm{B}} T}{\boldsymbol{e}}\right) y^{2} \frac{R_{\mathrm{S}}^{2}}{r^{2}} \ln \left(1+\exp \left(-\kappa\left(r-2 R_{\mathrm{S}}\right)\right)\right)
$$

Here, $\varepsilon$ is the dielectric constant of the solution, $k_{\mathrm{b}}$ is the Boltzmann constant, $T$ is the temperature, $\boldsymbol{e}$ is the elementary charge, and $y$ is the absolute surface potential. The Debye screening parameter is a function of valency and concentration that can be calculated from

$$
\kappa=\left(\frac{2 \rho \boldsymbol{e}^{2}}{\varepsilon k_{\mathrm{b}} T} \sum x_{i} z_{i}^{2}\right)^{1 / 2}
$$

Here $\rho$ is the salt concentration, $x_{i}$ and $z_{i}$ are the fraction and the charge of ion species of the $i$-th component, respectively.

Equation S8 necessitates the evaluation of $y$ strictly at the SiNP surface as opposed to the commonly used zeta potential. The absolute surface potential, until recently, has been challenging to measure. However, if one has an accurate surface charge density $q$ by solving the modified Grahame equation, ${ }^{12}$ namely

$$
q=\frac{2 \varepsilon k_{\mathrm{b}} T}{\boldsymbol{e} z} \kappa\left(2 \sinh \left(\frac{\Psi_{s}}{2}\right)+\frac{4}{\kappa R} \tanh \left(\frac{\Psi_{s}}{4}\right)\right) \quad \text { and }
$$




$$
\Psi_{s}=\frac{e z y}{2 k_{\mathrm{b}} T}
$$

The surface charge density for silica at various $\mathrm{pH}$ has been reported in several literature works. ${ }^{15}$ Here, we refer to the $q$ values for SiNP $<200 \mathrm{~nm}$ as suggested by the work of silica force field (FF) parameters that we use for our atomistic molecular dynamics (MD) simulations. ${ }^{15}$ We use a surface potential of $-170 \mathrm{mV}$ for $\mathrm{pH} 7.5$ and $-200 \mathrm{mV}$ for $\mathrm{pH} 8.5$ as input for Eq. S8.

As a self-consistent check, we can verify our computed values using the recent experimental measurements of Brown and co-workers, ${ }^{16}$ where the absolute surface potentials for $9 \mathrm{~nm}$ SiNP in various solutions are produced using X-ray photoelectron spectroscopy (XPS). From the figures provided by the paper, we extract $y$ in $50 \mathrm{mM} \mathrm{NaCl}$ aqueous solution at pH of 7.5 and 8.5 to be $\sim-145$ and $\sim-208 \mathrm{mV}$, respectively. The computed (based on $q$ ) and measured values of the surface potential are in good agreement and differences are likely due to error bars and different medium conditions. Nevertheless, for the purposes of being consistent at the atomistic and CG level of our model, we adopt computed surface potentials as our input for Eq. S8.

\section{Short-range molecular interaction}

\section{Configuration setup}

As discussed in the main text, our model is built upon the assumption that the protein, sfGFP::Car9-Car9, is bound to a SiNP at all times through the tail-tag Car9, and that assembly is driven by the interaction between the loop-tag Car9 and other SiNPs. In order to estimate the binding affinity of the loop-tag Car9 to another SiNP, we set up atomistic MD simulations to obtain the binding free energy. We approximate the SiNP surface using a silica slab that is periodic in the $\mathrm{x}$ - and $\mathrm{y}$-directions. We describe the $\mathrm{Si}, \mathrm{O}$ and $\mathrm{H}$ atoms in the silica slab using the INTERFACE FF (Ref. ${ }^{15}$ ). This FF work suggests to use amorphous silica surfaces for $\mathrm{SiNP}<200 \mathrm{~nm}$, and tune the $\mathrm{pH}$ value by changing the ionization state 
of the surface silanol groups. Starting from the amorphous silica slab with all Q3 (vicinal silanol) provided by the FF work, we alter the ionization state to $14.5 \%$ for $\mathrm{pH} 7.5$ and $18 \%$ for $\mathrm{pH} 8.5$.

We construct a free Car9 peptide following the sequence: DSARGFKKPGKR, with ACE and NME caps to neutralize the charge at the end groups, as shown in Figure S7. The structure of the peptide is relaxed in water with counter ions for 2 ns in NPT ensemble (v-rescale thermostat and Berendsen barostat) and 20 ns in NVT ensemble (Nosé-hoover thermostat) at $300 \mathrm{~K} .{ }^{17,18}$ The relaxed Car9 is flexible and does not show a secondary structure, which is consistent with previous studies. $^{2}$

(a)

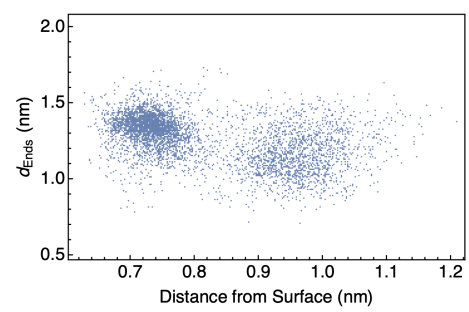

(b)

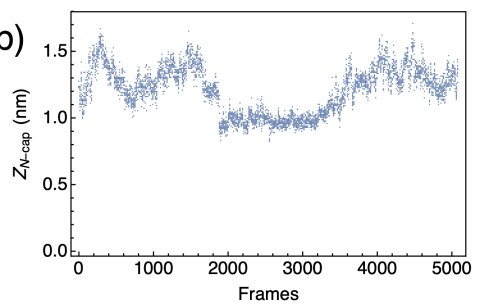

(c)

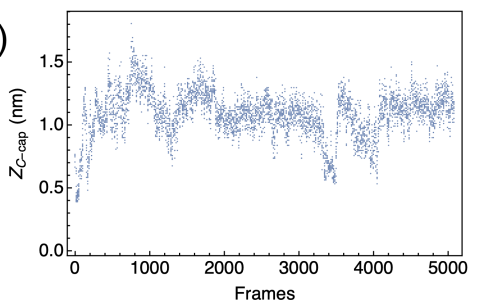

Figure S7: (a) Distribution of end-to-end distance of the loop-tag Car9 when fused in an sfGFP scaffold. (b) Location of the N-terminus of loop-tag Car9 in the simulation of sfGFP::Car9 on an amorphous silica slab. (c) Location of the C-terminus of loop-tag Car9 in the simulation of sfGFP::Car9 on an amorphous silica slab.

Since a free Car9 is flexible, whereas the loop-tag Car9 has both of its termini integrated within sfGFP, we constrain the end-to-end distance and end-to-surface height of a free Car9 orthogonal to the surface to make it "loop-tag-like". To acquire such information, we perform a simulation of an sfGFP::Car9 molecule on an amorphous silica slab, with the loop-tag Car9 oriented towards to the slab, an example configuration of which is as shown in Figure S8(c). Here, we focus on the end-to-end distance of the peptide and the end-to-surface between the two ends of the peptide and the surface, represented by the average $\mathrm{O}$ atoms position in the surface silanol groups. The end-to-end distance and end-to-surface distance distributions are shown in Figure S7. In our free energy calculations, we constrain the end-to-end distance between 0.8 and $1.8 \mathrm{~nm}$, and allow the lowest accessible end-to-surface distance to be 0.5 
(a)

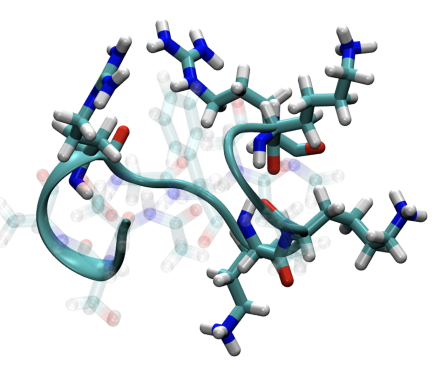

(b)

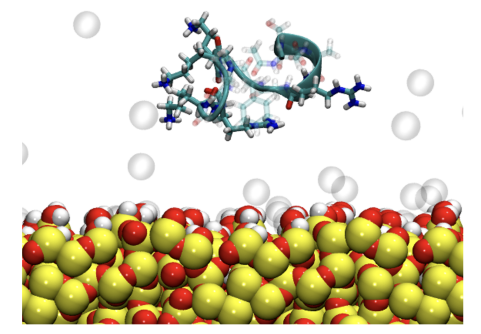

(c)

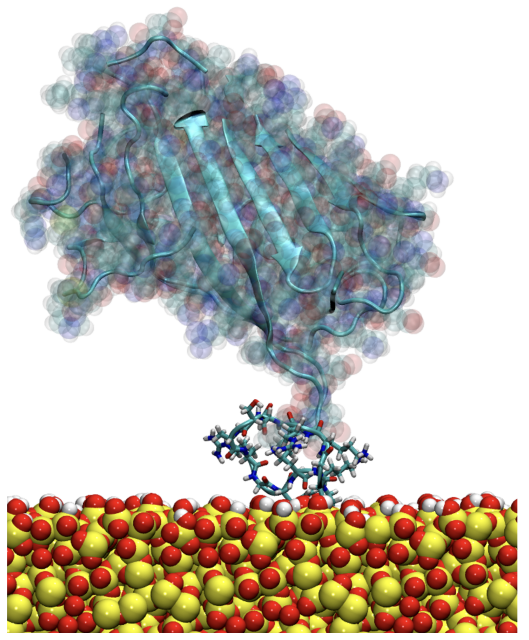

Figure S8: Configurations used in atomistic MD simulations. (a) Structure of the Car9 peptide, with positively charged residues (Arg and Lys) highlighted in licorice and other residues shown as transparent for simplification. (b) Example configuration for calculating the binding of Car9 to the silica surface. Solvent molecules (water) are ignored for clarity and $\mathrm{Na}$ ions are shown as clear spheres. (c) Example configuration of an sfGFP::Car9 molecule near the silica slab, with the loop-tag Car9 (highlighted in licorice) oriented towards the surface. Solvents and ions are removed for clarity.

nm.

\section{Parallel-bias metadynamics}

We use parallel-bias metadynamics (PBMetaD) with multiple walkers to calculate the binding energy of a loop-tag-like Car9 on amorphous silica surface at pH 7.5 and 8.5. We choose the average z-axis of $\mathrm{O}$ atoms in the surface silanol groups as the reference for the surface, and bias 14 low dimensional collective variables (CV) in parallel, namely, the z-component of the distance between the center of mass (COM) of the backbone relative to the surface, the z-component of the COM of the 12 amino acid residue head groups to the surface, and the radius of gyration. We deposit the same Gaussian hills $(\sigma=0.02 \mathrm{~nm}$ and height $=$ $1.2 \mathrm{~kJ} / \mathrm{mol}$ ) for all CVs every 1000 timesteps and use a bias factor of 20. An upper wall is placed $6.5 \mathrm{~nm}$ above the surface to limit the sampling space. In addition, for each walker, we start with Car9 at various locations and configurations to ensure sampling efficiency and avoid over-piling bias which may hinder the walkers from fully exploring the entire energy 
landscape.

For each $\mathrm{pH}$ value, our total sampling time is $4.8 \mu \mathrm{s}(1.2 \mu \mathrm{s}$ for each walker $)$ or till convergence. Once sampling is converged (as shown in Figure S9), we obtain the binding free energy profiles for the COM of Car9 on silica surface through standard re-weighting, and the free energy profile is shown in Figure 3 in the main text.

The PLUMED input files required to reproduce the results reported in this paper are available on PLUMED-NEST (www.plumed-nest.org), the public repository of the PLUMED consortium, ${ }^{19}$ as plumID:21.022

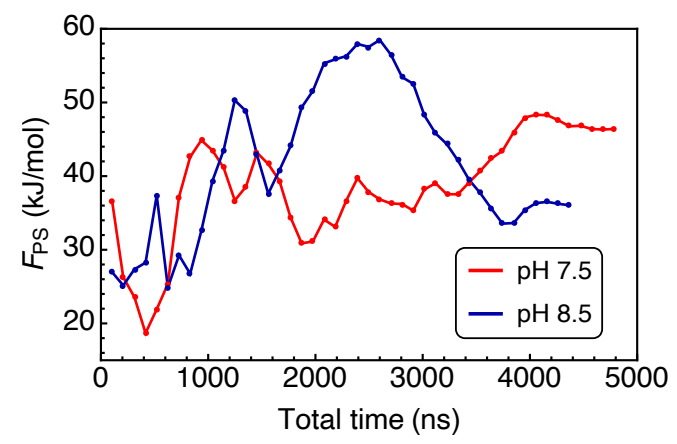

Figure S9: The binding free energy of a loop-tag-like Car9 on amorphous silica surface as a function of time.

\section{Effect of curvature}

We argue in the main text that approximating the interaction between the protein and SiNP using the binding free energy obtained for a flat surface largely overestimates the binding affinity, which will result in irreversible and $\mathrm{pH}$-independent assembly. This is because such approximation ignores the curvature effect, which is in fact significant when the dimension of the protein is comparable to that of the SiNP. We choose to employ the surface element integration (SEI) method to include the effect of curvature. This method has been shown to be resolve the over-correction problem in the Derjaguin approximation for the separation range $r-2 R_{\mathrm{S}} \sim O\left(R_{\mathrm{S}}\right){ }^{20}$

Since the driving force for bringing two particles together does not come from the SiNPs themselves, but from their surface decoration by proteins that we take to be irreversibly 
bound to the SiNPs through their tail Car9 sequence, we take an unconventional way to accommodate such scheme, as shown in Figure S10. We start with a configuration Figure S10(a) that is intrinsically implied when we obtain the protein-silica interaction from simulations with flat surface, where silica surfaces A and B approach each other driven by the attraction between $\mathrm{A}$ and $\mathrm{C}$ (i.e. the protein attached to $\mathrm{B}$ via the tail Car9). Since the protein is inseparable from B and the assembly can only happen when $\mathrm{C}$ is present, we can consider as a part of A-B interaction where we can use a per-area quantity A-C' interaction to describe the attraction, like one illustrated in Figure S10(b). Because each SiNP is decorated by an average of five proteins, we consider $1 / 5$ of the SiNP surface area to be an appropriate denominator to obtain the effective per-area interaction (i.e., the five proteins together contribute to a "uniform" distribution of the attractive driving force over the entire SiNP surface). The effective per-area attraction can be applied in the SEI to include the effect of curvature and allow the configurational set up to transit from Figure S10(b) to Figure S10(c), and the final expression obtained through SEI is no longer a "per-area" property, rather, it represents the effective attraction interaction between spheres A and B due to the presence of C'. Finally, to maintain the directionality of such attraction, we assign the "effective" interaction back on to the location of the attached protein and arrive at the expression in Figure S10(c), which serves as the configurational set up for our rigid-body model in the next step.

To transit from Figure S10(b) to (c), we follow the work by Bhattacharjee and cowokers. ${ }^{21}$ The total interaction between two spheres is

$$
U(H)=U_{12}^{A A}-U_{12}^{A^{\prime} A}-U_{12}^{A A^{\prime}}+U_{12}^{A^{\prime} A^{\prime}}
$$

in which, $H$ is the center-to-center distance of spheres. Each $U$ on the right hand side of Eq. S12 can be calculated using the following equations: in which, $a_{1}$ and $a_{2}$ are the radii of the two SiNPs, $r$ is the radius of the circular element being integrated as shown in Ref, ${ }^{21}$ 
(a)

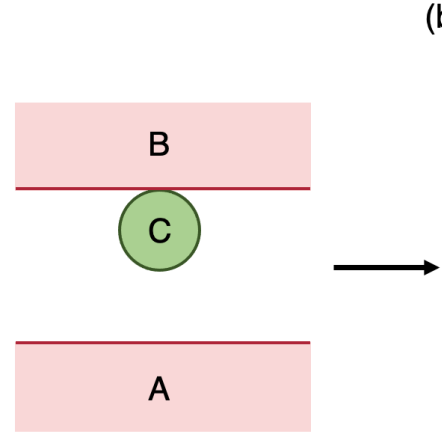

(b)
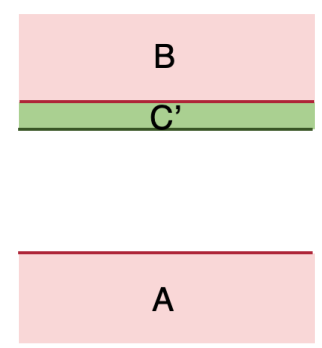

(c)
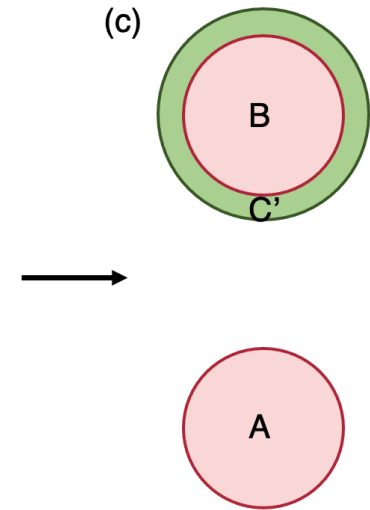

(d)
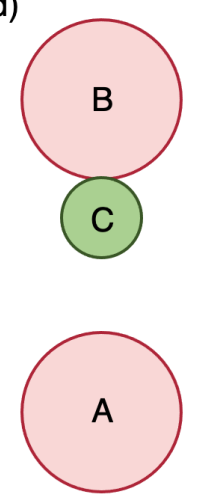

Figure S10: Illustrative cartoon for the scheme used to include the effect of curvature for protein-SiNP interaction. (a) The implied model for two silica surfaces (slabs A and B shown in pink) attracted to each other due to the presence of the protein (sphere C shown in green) when using a periodic flat surface to calculate the protein-silica interaction. (b) Conversion of the protein-silica interaction into a "per area" representation $\mathrm{C}$ ' in preparation for applying the SEI. (c) Consideration of the effect of curvature by employing the SEI. (d) Imposed attraction due to the presence of protein between a protein-carrying SiNP and another SiNP back on to the location where the protein is attached to recover the directionality of the interaction.

and $E$ is the "per-area" interaction on a flat surface. ${ }^{20}$

\section{Rigid-body simulation}

\section{Configuration setup}

In the rigid-body simulation, the initial configuration contains 100 singlets in a simulation box of $321.4 \mathrm{~nm} \times 321.4 \mathrm{~nm} \times 321.4 \mathrm{~nm}$. Each singlet is comprised of one large sphere

$$
\begin{aligned}
U_{12}^{A A} & =2 \pi \int_{0}^{a_{1}}\left(\sqrt{1-r^{2} / a_{2}^{2}}\right) E\left[H-a_{1} \sqrt{1-r^{2} / a_{1}^{2}}-a_{2} \sqrt{1-r^{2} / a_{2}^{2}}\right] r d r \\
U_{12}^{A^{\prime} A} & =2 \pi \int_{0}^{a_{1}}\left(\sqrt{1-r^{2} / a_{2}^{2}}\right) E\left[H+a_{1} \sqrt{1-r^{2} / a_{1}^{2}}-a_{2} \sqrt{1-r^{2} / a_{2}^{2}}\right] r d r \\
U_{12}^{A A^{\prime}} & =2 \pi \int_{0}^{a_{1}}\left(\sqrt{1-r^{2} / a_{2}^{2}}\right) E\left[H-a_{1} \sqrt{1-r^{2} / a_{1}^{2}}+a_{2} \sqrt{1-r^{2} / a_{2}^{2}}\right] r d r \\
U_{12}^{A^{\prime} A^{\prime}} & =2 \pi \int_{0}^{a_{1}}\left(\sqrt{1-r^{2} / a_{2}^{2}}\right) E\left[H+a_{1} \sqrt{1-r^{2} / a_{1}^{2}}+a_{2} \sqrt{1-r^{2} / a_{2}^{2}}\right] r d r
\end{aligned}
$$


representing the SiNP and few small spheres on the surface at randomized locations. The number of small spheres is set corresponding to the prot:SiNP molar ratio, for example, each large sphere carries 5 small spheres if prot:SiNP $=5: 1$. Then we divide the simulation box evenly into 125 cubes and insert the 100 particles at the center of 100 randomly selected cubes.

\section{Protein-protein interaction justification}

For the short range interaction, we only include the significant protein-SiNP attraction and assume that the protein-protein interaction is trivial compared to other interactions when particles are close. This assumption is based on two justifications. First, based on the fraction of SiNP surface area covered by proteins, the probability of two proteins from different particles interacting each other is negligible compared to the probability of a protein interacting with the SiNP surface. This can be justified by a simple probability argument based on surface area ratios. The protein is a beta barrel characterized by a diameter of $2.4 \mathrm{~nm}$ and a height of $4.2 \mathrm{~nm}$ with two Car9 inserted at the two ends of the cylinder, likely adopts a configuration as shown in Figure S11, with the axis perpendicular to the surface when attached on the SiNP surface. Because of this "standing" configuration, we approximate the surface area of the protein using the diameter of the "projected surface area," i.e. $2.4 \mathrm{~nm}$. The surface area ratio of protein and SiNP at prot:SiNP=5:1 results in a probability of $7.8 \%$ for a direct protein-protein contact, which is negligible comparing to other stronger interactions. Second, for proteins that do not have a propensity to oligomerize or cluster, protein-protein interactions can be neglected in front of the strong binding of the loop Car9 SBP to SiNP.

\section{Effect of potential switch on the rigid-body simulation results}

Although the $W_{\mathrm{SS}}$ between two colloids takes the form of $1 / \mathrm{r}$ as a result of summing up multiple "atomic" sites comprising the colloid, its scale decays fast due to the use of the Hamaker 


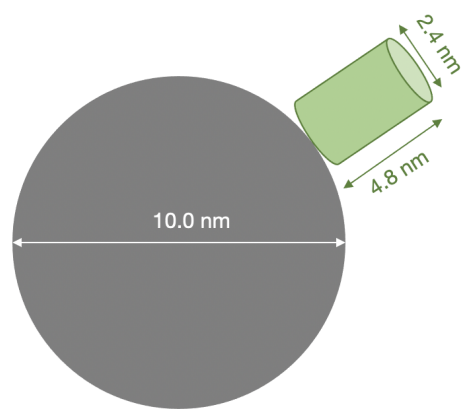

Figure S11: A cartoon illustration of a protein on an SiNP.

constant, which includes material-dependent information. Nevertheless, other interactions that adopts $\propto 1 / \mathrm{r}$ in MD simulations (e.g., electrostatic interaction) often require delicate treatment in long range interaction. Here, we demonstrate that, since the $W_{\mathrm{SS}}$ is already a fast decaying interaction, it is robust to various long-range treatments. We compare the $W_{\mathrm{SS}}$ used for production in this study to two other similar $W_{\mathrm{SS}}$ that are switched to the faster-decaying $1 / r^{6}$ at $30 \mathrm{~nm}$ and $100 \mathrm{~nm}$ cutoff, respectively. In Figure S12, we show that results for both $\mathrm{pH} 7.5$ and 8.5 nicely collapse to the trend characterized by our current results.

\section{Calculating I(q)}

We compare the $I(q)$ obtained from simulated with various prot:SiNP molar ratios to that from the USAXS to further narrow down the attractive range between sfGFP::Car9-Car9 and SiNP. The $I(q)$ is calculated as

$$
I(q)=F^{2}(q) S(q)
$$

in which, $F(q)$ is the form factor of each particle and $S(q)$ is the structure factor of the system. We approximate $F(q)$ using the form factor for a sphere via

$$
F(q)=\frac{3(\sin [q R]-q R \cos [q R])}{(q R)^{3}}
$$


(a)

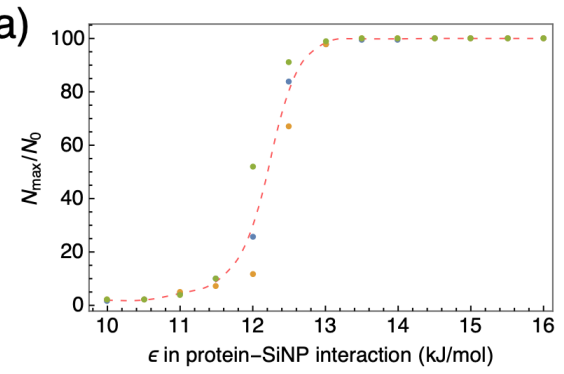

(c)

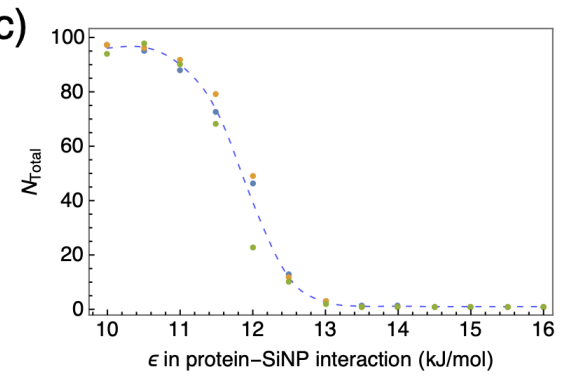

(b)

- No Switch

- Switch at $30 \mathrm{~nm}$

- Switch at $100 \mathrm{~nm}$

- No Switch

- Switch at $30 \mathrm{~nm}$

- Switch at $100 \mathrm{~nm}$

(d)
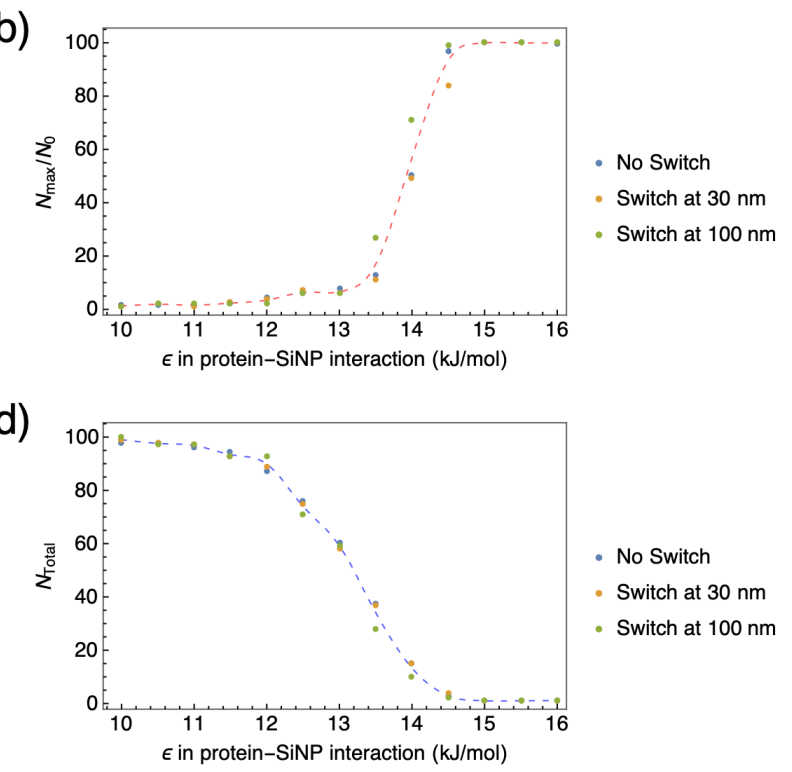

Figure S12: The state of assembly of the largest cluster for (a) $\mathrm{pH} 7$ and (b) $\mathrm{pH} 8$ with different potential switch schemes. The total number of clusters in the simulation box for (c) $\mathrm{pH} 7$ and (d) $\mathrm{pH} 8$ with different potential switch schemes.

where $R$ is the radius. The $S(q)$ can be obtained by Fourier transforming the radial distribution function $g(r)$ via

$$
S(q)=1+4 \pi \rho \int_{0}^{\infty} d r \frac{r^{2}(g(r)-1) \sin [q r]}{q r}
$$

where $\rho$ is the particle density and $r$ is the integrated distance in real space.

\section{I(q) at varying Prot:SiNP molar ratio and attraction at both $\mathrm{pH}$ values}

In addition to the selected computed $I(q)$ shown in the main text, we show all other $I(q)$ from simulation in Figure S13.

Here, we need to address the discrepancy and the modification to accommodate for the discrepancy that is mentioned in the main text. Note that in the simulated $I(q)$ in Figure $5(\mathrm{a})$ in the main text, the solid cyan curve (prot:SiNP $=1: 1$ ) mostly overlaps with the $I(q)$ for bare SiNP, and only the $I(q)$ of a system composed of $8 \mathrm{~nm}$ diameter SiNP with an average prot:SiNP $=1: 1$ together with all other $I(q)$ calculated with $10 \mathrm{~nm}$ SiNP will recover the trend 

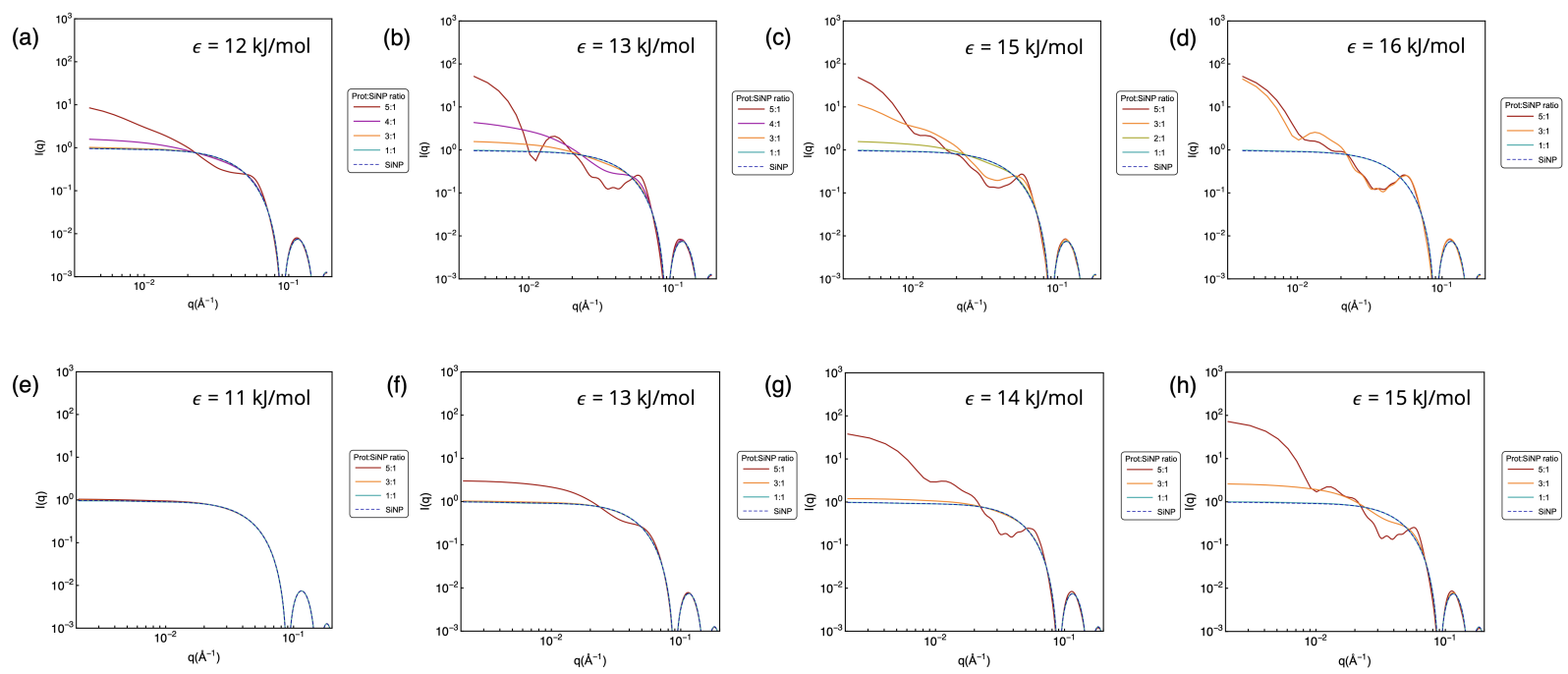

Figure S13: Computed $I(q)$ for varying prot:SiNP molar ratios at several $\epsilon$ values and at $\mathrm{pH}$ 7.5 ((a) to (d)) or at $\mathrm{pH} 8.5((\mathrm{e})$ to $(\mathrm{h}))$.

in experiment. We argue that the discrepancy is due to an artifact rather than to the model. In all other rigid-body simulations, we use a diameter of $10 \mathrm{~nm}$ for spheres representing the SiNP as indicated by the manufacturer. However, the distribution of SiNP sizes varies by batch and the mean diameter for the ones used in the USAXS experiment is measured to be $8.2 \mathrm{~nm}$, instead of $10 \mathrm{~nm}$. When the size of the SiNP decreases, the interparticle electrostatic repulsion will decrease due to the more significant effect of curvature. Therefore, the electrostatic repulsion in the sample used in the USAXS should be smaller than that we simulate, which requires weaker attraction to induce assembly. The discrepancy between the simulation and experiment should be significant when the prot:SiNP molar ratio is small, i.e., when the interplay between the repulsion and attraction is more delicate. Thus, to reproduce the trend in the experiment, we construct new systems with $8 \mathrm{~nm}$ SiNP for prot:SiNP=1:1.

In addition to the SiNP size, we also find it necessary to employ a polydisperse scheme for the 1:1 prot:SiNP molar ratio, which is a closer representation of the experimental system than a monodisperse scheme. Originally, and for for simplification purposes, we constructed a monodisperse rigid-body model with each particle carrying the same number of proteins. At the same time, we tested and confirmed that a polydisperse rigid-body model, where 
each SiNP may carry 2 to 6 proteins while the mean prot:SiNP=5:1, varies negligibly from the simplified system (data not shown). Yet, such an assumption gradually fails when the prot:SiNP molar ratio becomes very small (e.g., 1:1), since any variation in protein loading becomes significantly different from the mean value. Consequently, we populate $33 \%$ of the particles with no protein on their surface, $34 \%$ of the particles with one protein on their surface, and $33 \%$ of the particles with two proteins on their surface, to yield a system with a mean Prot:SiNP of 1.

By adopting the above well-justified modification, we successfully capture the key features of the USAXS data for $\mathrm{pH}$ 7.5. While such modification would also be proper at $\mathrm{pH} 8.5$, it is unnecessary for the purpose of matching USAXS data, since neither experiment, nor simulation indicate that assembly is occurring.

\section{Effect of system size on the state of assembly}

While our CG rigid-body simulation results qualitatively match well with the cluster sizes that are accessible in the USAXS experiments, we provide an additional set of the rigidbody simulations to test the robustness of our framework on system sizes. Here, we increase the system size to 800 protein-SiNP hybrid particles with 5:1 prot:SiNP molar ratios and perform the same set of simulations as in Figure 4. All simulations are run sufficiently long to ensure an equilibrium of cluster sizes and distributions.

Figure S14 shows that the simulations with a larger system size preserve the same trend as observed in Figure 4. Since small system is subjected to particle depletion, it is reasonable to see the values of $N_{\max } / N_{0}$ go beyond 100 in the larger system at stronger protein-SiNP attractions. The particle depletion also gives rise to the different $N_{\max } / N_{0}$ values at the $\epsilon$ at the sharp transition in Figure 4 (e.g., $\epsilon=12 \mathrm{~kJ} / \mathrm{mol}$ for $\mathrm{pH} 7.5$ ). 


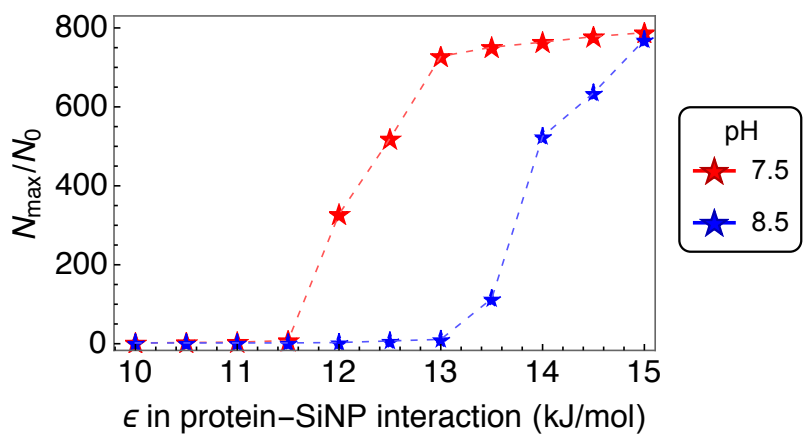

Figure S14: The assembled state $N_{\max } / N_{0}$ in systems of 800 rigid-body particles as a function of $\epsilon$ in the protein-SiNP interaction with 5:1 prot:SiNP molar ratio.

\section{Diffusivity}

The diffusivity in simulation is calculated from the slope of the mean-squared displacement (MSD), $\left\langle\left(r_{\mathrm{t}+\Delta \mathrm{t}}-r_{\mathrm{t}}\right)^{2}\right\rangle$, of a single SiNP in a simulation box. The final value is averaged over 50 individual simulations and each simulation is run for $50 \mathrm{~ns}$ under the NVT ensemble using the Nosé-Hoover chain thermostat at $300 \mathrm{~K} .^{22,23}$

For calculating the experimental diffusivity using the Stokes-Einstein equation, we use $1.0 \mathrm{cp}$ for the dynamic viscosity $\mu$, and $5 \mathrm{~nm}$ for $a$.

\section{Assembly kinetics}

A population balance equation can provide insight into assembly kinetics.

$$
J_{p}=\frac{d n_{p}}{d t}=\frac{1}{2} \sum_{\substack{i=1 \\ j=p-1}}^{p-1} k_{i, j} n_{i} n_{j}-\sum_{i=1}^{\infty} k_{i, p} n_{i} n_{p}
$$

describes the flux of a cluster of $p$ particles $J_{p}$ (i.e., $p$-mer) using the formation rate of smaller clusters (i.e. the first term) and the depletion rate to form larger clusters, and where $k$ is the rate constant. The flux in Eq. S17, based on the Smoluchowski's theory, can be used to estimate the stability ratio $W=J_{\text {ideal }} / J_{o b s}$, which describes how the observed assembly deviates from Brownian coagulation, where $J_{\text {ideal }}$ is the flux of Brownian aggregation in the absence of inter-particle interaction and $j_{o b s}$ is the observed flux. The observed assembly is 
reaction-limited when $W \gg 1$ and diffusion-limited if the opposite holds. With assumptions (i.e., 1) same size collision dominates, 2) $W_{i j}$ is size independent, and 3) the assembly is irreversible when solution condition does not change) ${ }^{24}$ we can utilize the very initial stage in the rigid-body simulation, where only singlets and doublets exist, to characterize the type of assembly kinetics. In this case, we focus on $W_{2}=J_{2, \text { ideal }} / J_{2, \text { obs }}$. Since there is no depletion of doublet to form larger clusters, $W_{2}$ is further reduced to a ratio of rate constants $W_{2}=k_{11, \text { ideal }} / k_{11, \text { obs }}$ from Eq. S17. The ideal $k_{11, \text { ideal }}$ for non-interacting particles is calculated using $8 k T / 3 \mu_{\text {sim }}$, with $\mu_{\text {sim }}$ being extracted from $D_{\text {sim }}$ and the Stokes-Einstein equation. The $k_{11, \text { obs }}$ can be estimated from the slope by plotting the number of doublet as a function of time $t$ as $\frac{1}{n_{2}}=\frac{1}{n_{o}}+k_{11, o b s} t .{ }^{12}$

Alternatively, based on the Smoluchowski's theory, $W$ can be extracted from the characteristic collision time $t_{c}$ by tracing the $p$-mer population as a function of time via

$$
n_{p}(t)=n_{0} \frac{\left(t / t_{c}\right)^{p-1}}{\left(1+t / t_{c}\right)^{p+1}} \quad t_{c}=\frac{3 \mu W}{4 n_{0} k_{b} T} .
$$

By utilizing the doublet formation as a function of time, we arrive at a $W_{2}=9.3$, which suggest a reaction-limited aggregation case. ${ }^{12}$

\section{Altering CG resolution}

To show the importance of the level of CG resolution to the validity of a physical model, we compare our model to an even more CG model with "angle-averaged" potential. As stated in the main text, the motivation to perform such an analysis is to understand the unprecedented induction time when each particle possesses relatively strong attraction to another particle ( 5 proteins with $\sim 4$ to $5 k_{b} T$ attraction each). Among the range of $\epsilon$ we scan for the protein-SiNP attraction, we choose to look more into the $\epsilon=12 \mathrm{~kJ} / \mathrm{mol}$ for $\mathrm{pH}$ 7.5. For more information, we provide the number of $p$-mer $(p \leq 15)$ and the number of particles in the largest cluster as a function of time in Figure S15 below. 


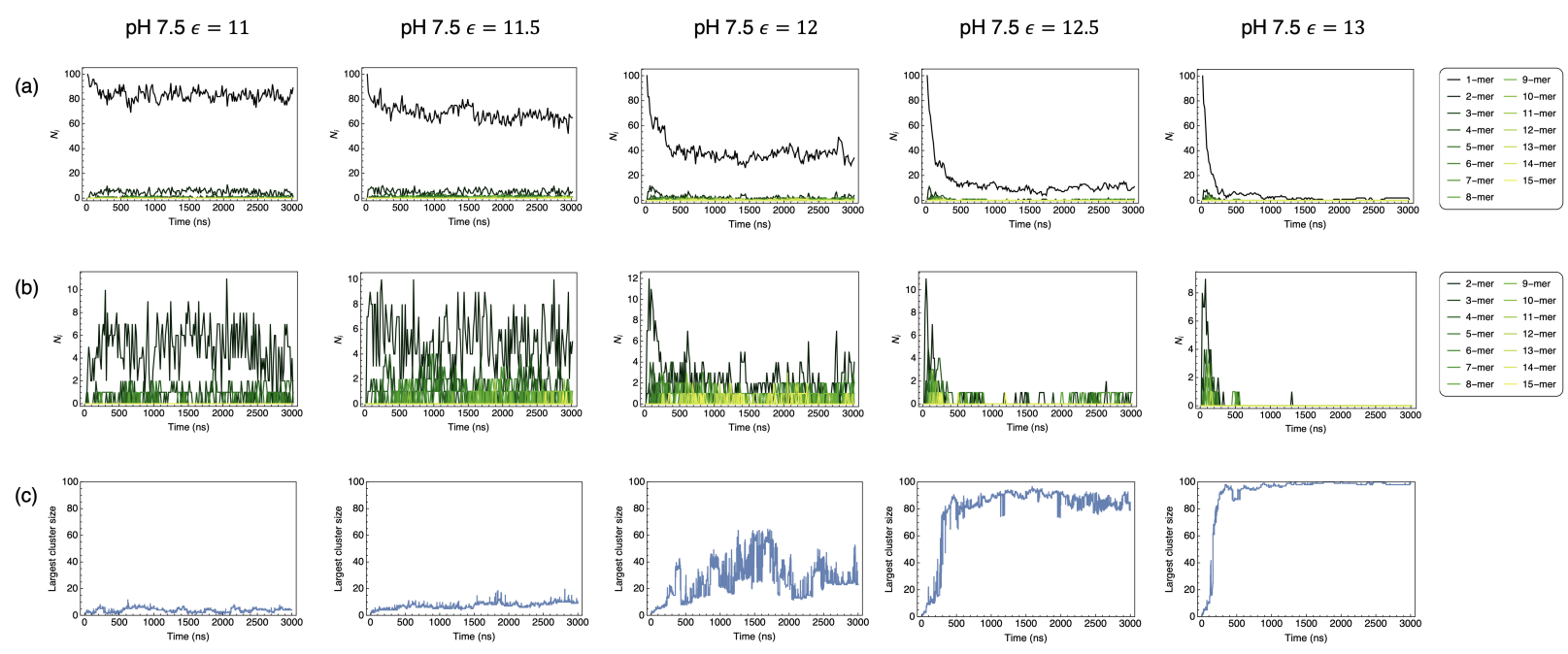

Figure S15: (a) Number of $p$-mer ( $p$ from 1 to 15) as a function of time, (b) a blow-up of (a) showing $p$-mer ( $p$ from 2 to 15), and (c) the number of particles in the largest cluster as a function of time in our rigid-body model.

The 'angle-averaged' potential of mean force (PMF) $W(r)$ for $\epsilon=12 \mathrm{~kJ} / \mathrm{mol}$ is acquired by inverting the radius distribution function, $g(r)$, from the highlighted steady-state region in Figure S16 via $W(r)=-k_{\mathrm{b}} T \ln g(r)$. Since the inverted PMF (Figure S16) represents the overall effect from both SiNP-SiNP repulsion and protein-SiNP attraction, it is then used as an interaction descriptor for a new simulation system with only 100 spheres (each sphere represents a protein-adsorbed SiNP particle) in a box. We show the number of $p$-mer $(p \leq$ 15) and the number of particles in the largest cluster as a function of time of the more CG system in Figure S17.

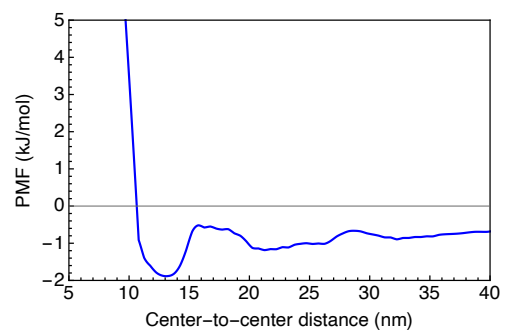

Figure S16: PMF obtained from the $\mathrm{g}(\mathrm{r})$ with $\epsilon=12 \mathrm{~kJ} / \mathrm{mol}$ at $\mathrm{pH} 7.5$

First, we notice a striking difference in assembly timescales between two CG resolutions, where the the new "angle-averaged" system quickly depletes all monomers within 3 ns, 
(a)

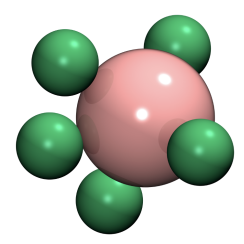

(b)

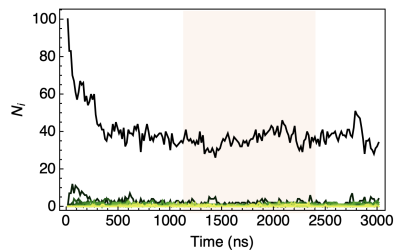

(c)

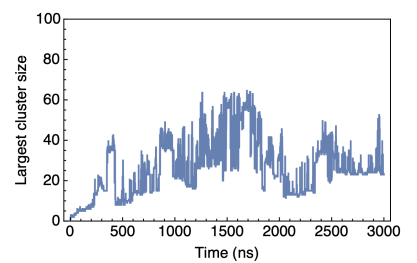

'Angle-averaged' model
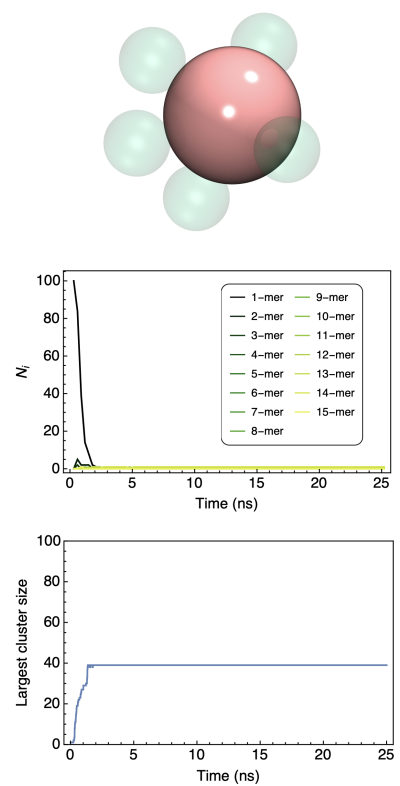

Figure S17: Comparison of the rigid-body model (left panel) and the "angle-averaged" model (right panel). (a) In our rigid-body model, both SiNP and protein are explicitly represented by pink and green spheres, respectively. In the 'angle-averaged' model the effect from the protein is averaged and imposed back onto the SiNP. (b) The number of $p$-mer $(p \leq 15)$ as a function of time. The highlighted region on the left panel indicates where the $\mathrm{g}(\mathrm{r})$ is selected. (c) Number of particles in the largest cluster as a function of time. 
whereas our original model reaches a steady-state around $500 \mathrm{~ns}$ and the system still remains relatively dispersed. The difference likely results from the directionality in attraction when proteins are explicitly represented.

We further notice that altering the CG resolution can also switch the assembly from reaction-limited aggregation to diffusion-limited aggregation, as we calculate the $W$ of the "angle-averaged" system to be 0.11 .

Therefore, since we have shown that our rigid-body model can correctly be scaled up to the characteristic experimental assembly timescale using the diffusivity, we conclude the importance of preserving the interaction directionality in CG models to maintain high fidelity to the experiments. 


\section{References}

(1) Hellner, B.; Lee, S. B.; Subramaniam, A.; Subramanian, V. R.; Baneyx, F. Langmuir 2019, 35, 5013-5020.

(2) Hellner, B.; Alamdari, S.; Pyles, H.; Zhang, S.; Prakash, A.; Sprenger, K. G.; Yoreo, J. J. D.; Baker, D.; Pfaendtner, J.; Baneyx, F. J. Am. Chem. Soc. 2020, 142, 2355-2363.

(3) Piston, D. W.; Kremers, G. J. Trends Biochem. Sci. 2007, 32, 407-414.

(4) Ilavsky, J.; Zhang, F.; Andrews, R. N.; Kuzmenko, I.; Jemian, P. R.; Levine, L. E.; Allen, A. J. J. Appl. Crystallogr. 2018, 51, 867-âĂŞ882.

(5) Ilavsky, J.; Jemian, P. R. J. Appl. Crystallogr. 2009, 42, 347-353.

(6) Doucet, M. et al. SasView. 2020.

(7) Doucet, M. et al. Sasmodels. 2020.

(8) Kienzle, P. A.; Krycka, J.; Patel, N.; Sahin, I. Bumps. University of Maryland: College Park, MD, 2011.

(9) Teixeira, J. J. Appl. Cryastallogr. 1988, 21, 781-785.

(10) Kline, S. R. J. Appl. Cryastallogr. 2006, 39, 895-900.

(11) Kim, A.; Berg, J. C. Langmuir 2000, 16, 2101-2104.

(12) Russel, W. B.; Saville, D. A.; Schowalter, W. R. Colloidal Dispersions, 1st ed.; Cambridge University Press, 1991.

(13) Bergström, L. Adv. Colloid Interface Sci. 1997, 70, 125-169.

(14) Sader, J. E.; Carnie, S. L.; Chan, D. Y. C. J. Colloid Interface Sci. 1995, 171, 46-54. 
(15) Emami, F. S.; Puddu, V.; Berry, R. J.; Varshney, V.; Patwardhan, S. V.; Perry, C. C.; Heinz, H. Chem. Mater. 2014, 26, 2647-2658.

(16) Brown, M. A.; Abbas, Z.; Kleibert, A.; Green, R. G.; Goel, A.; May, S.; Squires, T. M. Phys. Rev. X 2016, 6, 011007.

(17) Nosé, S. J. Chem. Phys. 1984, 81, 511-519.

(18) Hoover, W. G. Phys. Rev. A 1985, 31, 1695-1697.

(19) The PLUMED consortium, Nat. Methods 2019, 116, 670-673.

(20) Bhattacharjee, S.; Elimelech, M. J. Colloid Interface Sci. 1997, 193, 273-285.

(21) Bhattacharjee, S.; Elimelechand, M.; Borkovec, M. Croat. Chem. Acta. 1998, 71, 883903.

(22) Martyna, G. J.; Klein, M. L.; Tuckerman, M. J. Chem. Phys. 1992, 97, 2635-2643.

(23) Qi, X.; Balankura, T.; Zhou, Y.; Fichthorn, K. A. Nano letters 2015, 15, 7711-7717.

(24) Lee, J.; Nakouzi, E.; Song, M.; Wang, B.; Chun, J.; Li, D. ACS Nano 2018, 12, $12778-12787$. 